\title{
PRIMARY CUTANEOUS NOCARDIOSIS SECONDARY TO NOCARDIA ASTEROIDES: AN UNUSUAL PRESENTATION AT A RARE SITE
}

Partha Guchhait ${ }^{1}$, Gayathri Devi², Praveen Kumar S3, Kavitha Karur ${ }^{4}$, Peralam Yegneswaran Prakash ${ }^{5}$

\section{HOW TO CITE THIS ARTICLE:}

Partha Guchhait, Gayathri Devi, Praveen Kumar S, Kavitha Karur, Peralam Yegneswaran Prakash. "Primary Cutaneous Nocardiosis Secondary to Nocardia Asteroides: An Unusual Presentation at a Rare Site". Journal of Evolution of Medical and Dental Sciences 2014; Vol. 3, Issue 39, August 28; Page: 9870-9875,

DOI: $10.14260 /$ jemds/2014/3277

ABSTRACT: Primary cutaneous nocardiosis can present as an abscess or cellulitis with sporotrichoid morphology. Deep inoculation in the subcutaneous tissue can present as a mycetoma. Mycetoma over the elbow region is rare. We herein describe a case of primary cutaneous nocardiosis presenting as a mycetoma, caused by Nocardia asteroides. The patient had extensive keloidal lesions with few sinuses extruding serous discharge over the right forearm around elbow joint. Initially pus and later, the deep tissue biopsy samples were sent for culture and sensitivity, without suspecting nocardial infection. Gram stain of the pus showed few WBCs and occasional Gram positive branching filaments. Modified Kinyoun stain of the same revealed acid-fast filamentous structures. Culture of the tissue specimen yielded Nocardia asteroides. The patient responded well to a modified Ramam's regime. On follow-up, the patient showed no recurrence at 1 year. Our case demonstrates the need for focused clinical search with a high degree of suspicion, and appropriate laboratory procedures in the diagnosis and management of cutaneous nocardiosis.

KEYWORDS: Primary Cutaneous, Nocardial Mycetoma, Modified Ramam regimen.

INTRODUCTION: Nocardiosis is caused by several species of soil-borne aerobic bacteria belonging to the genus Nocardia. Cutaneous nocardiosis can be classified into Primary cutaneous nocardiosis and Secondary cutaneous nocardiosis (usually after dissemination from the lungs). Primary cutaneous nocardiosis is relatively rare. Three types of Primary cutaneous nocardiosis have been identified: an acute superficial infection with abscess or cellulitis, a lymphocutaneous (sporotrichoid) infection and a deeper infection, mycetoma.

None of these three types possess any characteristic feature that would make a definitive clinical diagnosis possible. [1] Pinoy, in 1913, subdivided the mycetomas into two groups: one caused by Eumycetes or "true fungi", Eumycetoma and other by aerobic filamentous bacteria belonging to class "Actinomycetes" named Actinomycetoma. Mycetoma is characterized by the triad of tumefaction of affected tissue, multiple discharging sinuses, and the presence of oozing granules. [2] The genus Nocardia belongs to the order Actinomycetales, a group of aerobic, Gram-positive filamentous bacteria.

The organism is geophillic and is found in soil and decaying plant parts. Isolation and species identification of nocardia from different clinical specimens is difficult and needs the expertise of a microbiologist. An early diagnosis and treatment of Nocardial mycetoma prevents the need for extensive surgical debridement and complications. ${ }^{[2]}$ Here, we report a case of mycetoma caused by Nocardia asteroides, presenting with an unusual keloidal morphology over the right elbow joint, which showed a dramatic response to a modified Ramam's regime. 
CASE REPORT: A 25 years old female, mason by occupation, presented with an 8 year history of hyper pigmented dark brown plaque, resembling a keloid over the right forearm, around elbow joint. It was initially small in size, and then progressed gradually to cover the entire right elbow joint. There was history of itching over the lesion. No history of trauma was elicited. There was no history of chronic illness such as diabetes, tuberculosis or malignancy. On examination, lesion was indurated, irregular in shape with undefined borders, non-tender with few sinuses extruding serous discharge. No granules were noted in the discharge (Fig. 1).

Provisional diagnosis on the basis of above findings was made as keloidal lobomycosis, mycetoma, lupus vulgaris and atypical mycobacterial infection. A KOH mount from the discharge did not show any fungal elements. Gram stain of the pus showed long, beaded Gram-positive branching filaments (Fig. 2). Modified Zeil-Neelsen stain revealed weakly acid-fast structures. The skin biopsy sample of deeper tissue was sent for histopathological examination, aerobic culture, AFB culture and fungal culture. The specimen was inoculated in Blood agar, MacConkey agar, Lowenstein-Jensen media and Sabouraud's dextrose agar. ${ }^{[3]}$

Culture on Lowenstein-Jensen media showed dry, granular, wrinkled, yellowish-orange coloured colonies after 3 weeks (Fig. 3). Powdery white granular wrinkled colonies were found in Sabouraud's dextrose agar media after 6 weeks (Fig. 4). Gram's stain from the growth showed Gram positive long, beaded, branching filaments (Fig.5) and Modified Kinyoun's stain (using $1 \% \mathrm{H}_{2} \mathrm{SO}_{4}$ ) revealed long, branching, filamentous, partially acid fast bacilli morphologically resembling Nocardia species (Fig. 6). A positive urease test, growth at $42^{\circ} \mathrm{C}$ and inability to hydrolyse casein, tyrosine and xanthine confirmed the growth as Nocardia asteroides. ${ }^{[4]}$

Antibiotic sensitivity test by disc diffusion method showed the isolate to be sensitive to Cotrimoxazole $(1.25 / 23.75 \mu \mathrm{g})$, Tetracycline $(30 \mu \mathrm{g})$ and resistant to Penicillin (10units), Ampicillin $(10 \mu \mathrm{g})$, and Erythromycin $(10 \mu \mathrm{g})$. The isolate was sent to Kasturba medical college, Manipal for species reconfirmation and was identified as Nocardia asteroides. Histopathological examination of skin biopsy showed features of acute suppurative foci with few micro-abscesses. X-ray of right elbow joint (AP and Lateral view) was normal.

The patient was started on modified Ramam's regime. [5] There was significant clinical improvement with marked reduction in swelling and sinuses after 3 months of treatment. Patient was subjected to a baseline hemogram, routine urine analysis, renal function test, liver function test and a pure tone audiometry before every cycle of Ramam's regime. The Patient was treated with 4 cycles of this regime. There was near complete regression of the plaque and healing of the sinuses, with no recurrence after 1 year of follow-up (Fig. 7).

DISCUSSION: Nocardial infections can occur worldwide, particularly in tropical and subtropical countries. In India, the incidence of Nocardial mycetoma varies from $5.2 \%$ to $35 \%$ of all mycetomas. Mycetoma is the most common presentation of Primary cutaneous nocardiosis. Among the several species of Nocardia causing cutaneous infections, Nocardia brasiliensis is the most common species isolated followed by Nocardia asteroides, which usually causes fulminant systemic infection.

Other pathogenic species like N. transvalensis, N. otitidis-caviarum, and the recently recognized species $\mathrm{N}$. farcinica and $\mathrm{N}$. nova are rare causative agents for Primary cutaneous nocardiosis. [1] Our patient presented with hyper pigmented, dark brown plaque with few discharging 
sinuses of chronic duration, typical of Mycetoma. Nocardial mycetoma differs from other mycetoma by being not fibrotic and may present without grain formation.

The mycetoma usually occurs by introduction of its causative agent present in saprotrophic soil source into subcutaneous tissue, probably by accidental trauma by thorns or splinter injury. Adult males, especially bare-foot walkers, are the common sufferers of mycetoma. ${ }^{11}$ Surprisingly, our patient gave no history of injury.

Nocardia is an opportunistic pathogen, producing infection in the patients who are immunocompromised or have chronic debilitating disease and in solid organ transplant recipients. ${ }^{6]}$ In contrast, our patient was an immunocompetent healthy woman without any history of a chronic debilitating illness.

Identification and characterization of Nocardia species by culture is a tedious process as the organism may take up to 2 to 3 weeks for isolation from a clinical specimen and colonies being small may be overgrown by other rapidly growing organisms which results in an initial negative culture report for Nocardia.[7]

Culture takes long time but Gram stain of the pus gave an early indication towards Nocardiosis in our case and hence very important before other investigations are requested.

Cotrimoxazole forms the mainstay of therapy. In systemic form of disease, other drugs such as amikacin, gentamicin, dapsone, cephalosporins, minocycline, erythromycin, ciprofloxacin, imipenem and clindamycin can be used.

One to four months of therapy is usually curative for superficial cutaneous nocardiosis whereas mycetoma may need prolong treatment. ${ }^{[8]}$ Our patient responded well to "Modified Ramam regime", a two-step regimen, with an intensive phase (step-1) with gentamycin, $80 \mathrm{mg}$ IV 12 hourly and cotrimoxazole $80 / 400 \mathrm{mg}$ two tablets twice daily for a month, followed by step 2 with cotrimoxazole $80 / 400 \mathrm{mg}$ two tablets twice daily and 100mg doxycycline twice daily until all sinuses are healed.[9]

CONCLUSION: Primary cutaneous nocardiosis remains a diagnostic challenge. A high index of clinical suspicion along with the stringent efforts of microbiologist is required to diagnose and isolate the causative organism. Timely initiation of effective therapy is crucial for a favorable outcome. With a rich clinical expertise of usages of these drugs, which have a synergistic effect, with an additional advantage of being cost-effective, provided a baseline to use this two-step modified regimen in the successful management of our case.

\section{REFERENCES}

1. Inamadar AC, Palit A. Primary cutaneous nocardiosis: A case study and review. Indian J Dermatol Venereol Leprol 2003; 69 (6): 386-91.

2. Praveen kumar s et al. An unusual presentation of primary cutaneous nocardiosis at a rare site: successful treatment with a modified Welsh regimen. Dermatol Online J. 2011; 17 (12): 1.

3. Elmer K, Stephen A, William J, Paul S, Washington W. Koneman's; 'Colour Atlas and Textbook of Diagnostic Microbiology', 5 th ed. Philadelphia Lippincott Williams and Wilkins; 1997: 693-694.

4. Kiska DL, Hicks K, Pettit DJ. Identification of medically relevant Nocardia species with an abbreviated battery of tests. Journal of Clinical Microbiology 2002; 40 (4): 1346-1351. 
5. Ramam M, Ramkrishna B, Taru G et al. A modified two-step treatment for actinomycetoma. Indian J Dermatol Venereol Leprol 2007; 73: 235-9.

6. Baradkar VP, Mathur M, Kulkarni SD, Kumar S. The sporotrichoid pattern of cutaneous Nocardia asteroids. Indian J Pathol Microbiol 2008; 51: 432-34.

7. Sreeja S Beena, Banashankari G S, Rudresh H K. Nocardial Mycetoma: Re-infection with a different species at an unusual site - A rare case. Indian J Pathol Microbiol 2013; 56 (3): 333-34.

8. Vikrant A Saoji, Sandhya V Saoji, Rutuja W Gadegone, Priyanka R Menghani. Primary Cutaneous Nocardiosis. Indian Journal of Dermatology 2012; 57 (5): 404-06.

9. U S Agarwal, R K Besarwal, R Gupta, P Agarwal. Treatment of actinomycetoma foot - our experience with ten patients. JEADV 2013; 27, 1505-13.

Fig. 1: Hyperpigmented dark brown plaque with few discharging sinuses (On admission).

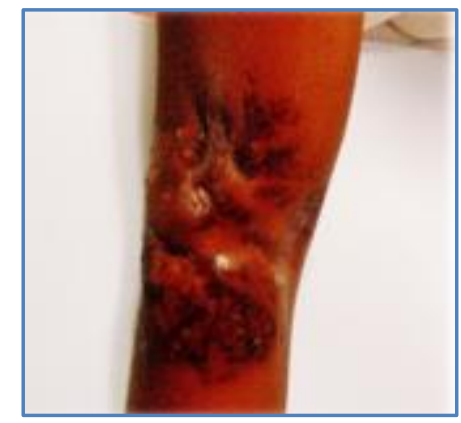

Fig. 1

Fig. 2: Gram stain from the pus sample showing pus cells and long Gram positive branching filaments.

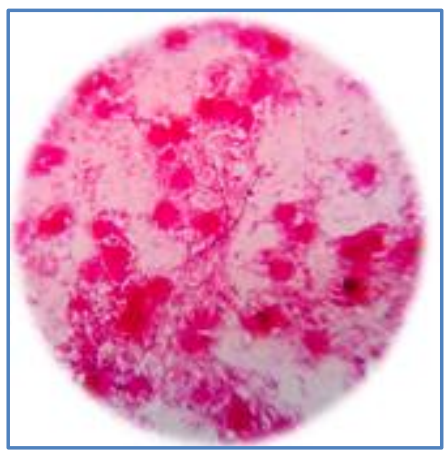

Fig. 2 
Fig. 3: Growth of Nocardia asteroides on Lowenstein-Jensen medium.

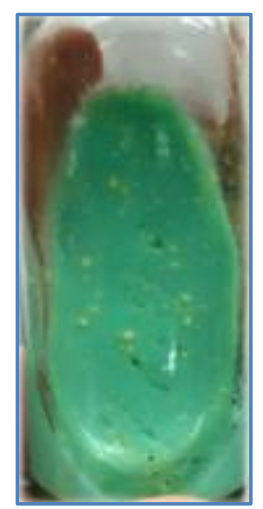

Fig. 3

Fig. 4: Growth of Nocardia asteroides on Sabouraud's Dextrose Agar medium.

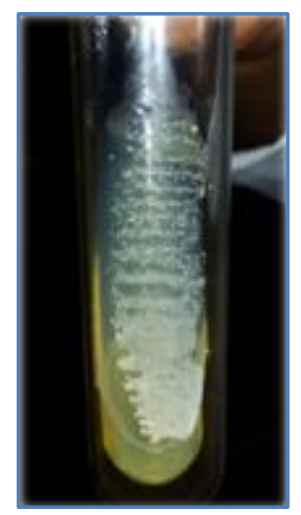

Fig. 4

Fig. 5: Gram stain from growth on Lowenstein-Jensen medium.



Fig. 5 
Fig. 6: Kinyoun staining shows thin long branching weakly acid-fast filaments.

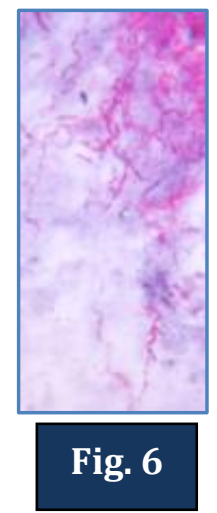
scarring.

Fig. 7: Lesions at 1year of follow-up showed complete healing of sinuses and hyperpigmented



\section{Fig. 7}

\section{AUTHORS:}

1. Partha Guchhait

2. Gayathri Devi

3. Praveen Kumar S

4. Kavitha Karur

5. Peralam Yegneswaran Prakash

\section{PARTICULARS OF CONTRIBUTORS:}

1. Post Graduate Cum Tutor, Department of Microbiology, M. S. Ramaiah Medical College and Teaching Hospital.

2. Professor, Department of Microbiology, M. S. Ramaiah Medical College and Teaching Hospital.

3. Assistant Professor, Department of Dermatology, M. S. Ramaiah Medical College and Teaching Hospital.

4. Post Graduate Cum Tutor, Department of Microbiology, M. S. Ramaiah Medical College and Teaching Hospital.
5. Assistant Professor, Department of Microbiology, Kasturba Medical College, Manipal.

\section{NAME ADDRESS EMAIL ID OF THE CORRESPONDING AUTHOR:}

Dr. Partha Guchhait, \#237, $5^{\text {th }}$ Main, $9^{\text {th }}$ Cross,

Pipeline Road, Mathikere, MSR Nagar, Bangalore-560054,

Karnataka.

Email: drparthaguchhait5@gmail.com

Date of Submission: $12 / 08 / 2014$.

Date of Peer Review: 13/08/2014.

Date of Acceptance: 22/08/2014.

Date of Publishing: 26/08/2014. 